\title{
Grau de confiabilidade na reprodução do planejamento virtual para o posicionamento final de implantes por meio de cirurgia guiada: relato de caso
}

\author{
Degree of reliability in the reproduction of virtual planning for final \\ placement of implants through guided surgery: case report
}

Karine Conte Brugnerotto Nuss*

Fernando Vacilotto Gomes ${ }^{* *}$

Frederico Mattis ${ }^{* * *}$

Luciano Mayer ${ }^{* * * *}$

\section{Resumo}

Introdução: na implantodontia atual, a precisão do planejamento pré-operatório, tanto durante a instalação dos implantes quanto no momento da restauração protética, é um pré-requisito fundamental para o sucesso da reabilitação do complexo maxilomandibular. Assim, as guias cirúrgicas para instalação dos implantes tornaram-se uma ferramenta valiosa para a transferência precisa do planejamento da posição dos implantes para o leito cirúrgico. A instalação de implantes por meio de cirurgias guiadas por guias prototipados apresenta elevadas taxas de sucesso, tendo como característica principal a redução do tempo operatório devido ao planejamento e à cirurgia virtual prévia. Objetivo: o objetivo deste trabalho é descrever as etapas técnicas de uma cirurgia guiada sem retalho em maxila edêntula de paciente, relatando um caso clínico em que se compara o planejamento virtual com o posicionamento final dos implantes instalados por meio de tomografias de feixe cônico. Relato de caso: paciente do gênero feminino, 64 anos, com desejo de realizar implantes em maxila edêntula, tendo indicação e disponibilidade óssea para realização de cirurgia guiada. Considerações finais: de acordo com o caso clínico apresentado, pode-se concluir que esta técnica cirúrgica permite um melhor pla- nejamento dos implantes, torna o procedimento cirúrgico mais preciso e rápido, como também aumenta a previsibilidade dos resultados.

Palavras-chave: Cirurgia assistida por computador. Implantes dentários. Prótese dentária fixada por implante.

\section{Introdução}

A quantidade e a qualidade do tecido ósseo remanescente deixaram de ser o fator primordial no planejamento cirúrgico para instalação de implantes osseointegráveis ${ }^{1}$. O planejamento reverso tendo como objetivo final a resolução protética é essencial para reabilitações sobre implantes com estética e função adequadas. Um planejamento pré-operatório preciso, tanto para instalação do implante quanto para confecção da restauração protética, é um pré-requisito fundamental para o sucesso da reabilitação oral de pacientes com implantes osseointegráveis ${ }^{1}$. Além disso, as tendências atuais concentraram-se em procedimentos cirúrgicos menos in-

Cirurgiã-dentista. Aluna do Curso de Especialização em Implantodontia da Associação Gaúcha de Ortodontia, Porto Alegre, RS, Brasil.

Cirurgião-dentista, Cirurgião e Traumatologista Bucomaxilofacial. Mestre em CTBMF pela Universidade Federal do Rio Grande do Sul, Porto Alegre, RS, Brasil. Aluno do Curso de Especialização em Implantodontia da Associação Gaúcha de Ortodontia, Porto Alegre, RS, Brasil.

*** Cirurgião-Dentista. Aluno do Curso de Especialização em Implantodontia da Associação Gaúcha de Ortodontia, Porto Alegre, RS, Brasil.

${ }^{* * *}$ Cirurgião-dentista, Implantodontista. Doutor em CTBMF pela Pontifícia Universidade Católica do Rio Grande do Sul, Porto Alegre, RS, Brasil. Coordenador do Curso de Especialização em Implantodontia da Associação Gaúcha de Ortodontia, Porto Alegre, RS, Brasil. 
vasivos e na redução do tempo de osseointegração ${ }^{2}$. Procedimentos que utilizem a cirurgia baseada em planejamento virtual inicial, em que este planejamento é transferido para o leito operatório "real" por meio de um guia prototipado, trazem vantagens significativas ao cirurgião-dentista e ao paciente ${ }^{2,3}$. Tais considerações versam sobre uma cirurgia sem retalho, na qual a redução do trauma e do tempo cirúrgico é muito significativa ${ }^{3}$.

A utilização de imagens virtuais geradas a partir de exames tomográficos para o planejamento e a colocação de implantes osseointegráveis é uma realidade na rotina clínica do implantodontista ${ }^{3}$. O diagnóstico por meio de tomografia computadorizada cone beam (TCCB) tornou-se amplamente acessível, pois fornece imagens tridimensionais (3D) das estruturas anatômicas, permitindo a aquisição de dados adequados, com uma dose de radiação relativamente baixa ${ }^{4}$. A TCCB é uma técnica precisa, não invasiva, que auxilia o profissional no diagnóstico e planejamento para o correto posicionamento dos implantes, especialmente quando utilizada em combinação com os softwares de planejamento ${ }^{2,5}$. Recentes avanços de imagens 3D em Odontologia, em combinação com a introdução de programas de planejamento para a inserção de implantes associada à instrumentação cirúrgica guiada, revolucionaram o diagnóstico e o tratamento com implantes dentários, além de criar um ambiente interdisciplinar em que a comunicação leva a uma melhor assistência, um melhor entendimento e melhores resultados para o paciente ${ }^{6}$.

A cirurgia virtualmente guiada associada a um planejamento computadorizado e realizada por meio de guias cirúrgicos prototipados representa um dos grandes avanços da implantodontia moderna ${ }^{4}$. Esse sistema de planejamento virtual permite a visualização das relações entre o posicionamento cirúrgico do implante a ser instalado e o posicionamento protético da reabilitação que será confeccionada, percebendo, antecipadamente, a necessidade de alterações no planejamento cirúrgico, podendo-se evitar o uso de intermediários angulados para compensar eventuais inclinações desfavoráveis dos implantes ${ }^{4-6}$. Devido a essas tecnologias, é possível predeterminar a posição tridimensional precisa do implante planejado antes da sua inserção real no leito cirúrgico ${ }^{1}$.

Para obtenção de uma guia para cirurgia guiada de implantes múltiplos, alguns passos são necessários, entre eles, a realização de uma TCCB do paciente, utilizando uma guia tomográfica, uma segunda aquisição apenas da guia tomográfica e, posteriormente, o planejamento, propriamente dito, em softwares específicos que combinam as imagens dos arquivos DICOM (Digital Imaging and Communications in Medicine) previamente obtidas. Esse protocolo, sendo rigidamente respeitado, possibilita ao cirurgião-dentista a confecção de uma prótese provisória antes mesmo da realização da cirurgia, permitindo a sua instalação imediatamente após a finalização do procedimento ${ }^{1,5-7}$.

Dessa forma, o objetivo deste trabalho é descrever as etapas técnicas da cirurgia guiada de uma paciente com maxila edêntula, relatando um caso clínico em que se compara o planejamento virtual (baseado na tomografia pré-operatória) com o aspecto da tomografia pós-operatória final, buscando-se verificar o real posicionamento dos implantes antes e após a cirurgia (virtual vs. real).

\section{Caso clínico}

Paciente do gênero feminino, 64 anos, compareceu no Curso de Especialização em Implantodontia da Associação Gaúcha de Ortodontia (Agor) a fim de substituir sua prótese total superior convencional por uma reabilitação implanto-suportada. Após a realização da anamnese, dos exames clínicos, da análise dos exames tomográficos e a verificação das relações intermaxilares, a paciente foi orientada quanto às vantagens de uma cirurgia guiada (Figura 1).

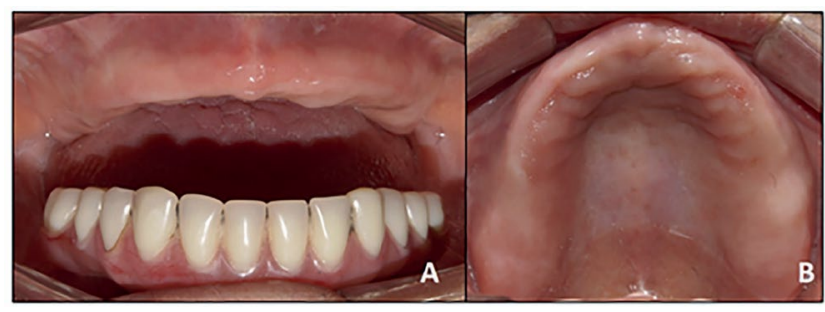

Figura 1 - Aspecto clínico inicial intrabucal da paciente: A) Vista frontal da maxila edêndula total e B) Vista oclusal do rebordo alveolar maxilar e palato duro

Fonte: elaboração dos autores.

Ao exame clínico, a paciente apresentava prótese total superior desadaptada, sendo que, no arco antagonista, fazia uso de um protocolo Branemark realizado há cerca de um ano. Para o refinamento do planejamento pré-operatório, realizou-se uma nova prótese total superior respeitando-se todos os preceitos protéticos, no intuito de reestabelecer uma adequada dimensão vertical e demais condições funcionais e estéticas de uma reabilitação removível. Posteriormente, foram realizadas perfurações com broca esférica de $2 \mathrm{~mm}$ na prótese total, as quais foram preenchidas com guta-percha para gerar marcas hiperdensas durante a captação da imagem tomográfica e, assim, demarcar os pontos na flange da prótese total (Figura 2). 


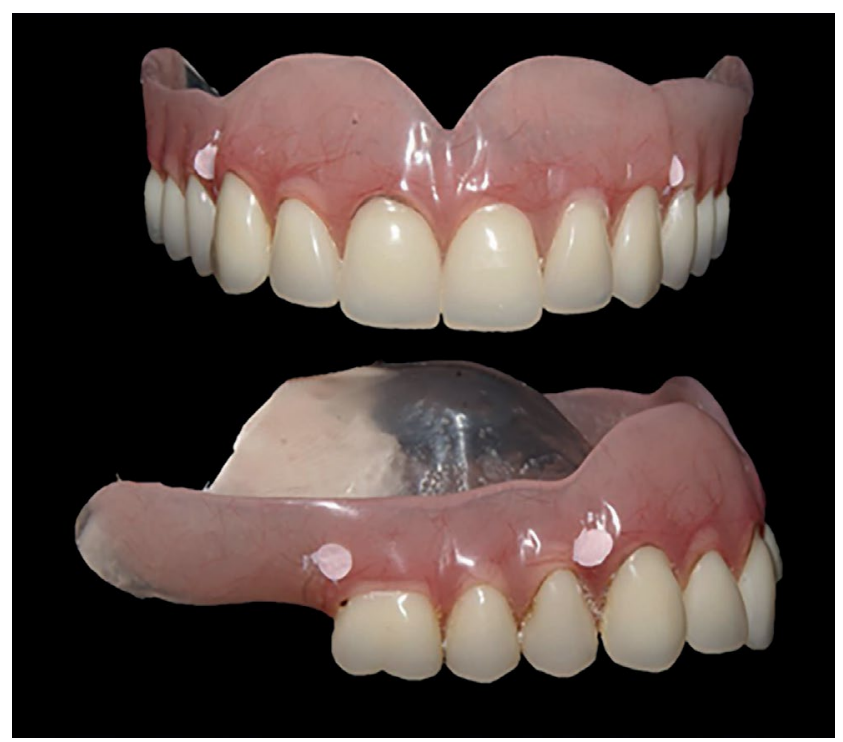

Figura 2 - Guia tomográfico: vistas frontal e lateral da prótese total da paciente que recebeu perfurações na flange acrílica e o preenchimento com guta-percha

Fonte: elaboração dos autores.

Durante a aquisição das imagens tomográficas, foi confeccionado e utilizado pela paciente, também, um registro interoclusal $(5 \mathrm{~mm})$ com silicone de adição pesado, a fim de manter o guia em uma posição ideal e estável.

Além das imagens tomográficas da paciente com o guia tomográfico em posição, foi realizada a tomografia da prótese total com as marcações de guta-percha (técnica do duplo escaneamento) com a finalidade de facilitar ao técnico em radiologia a sobreposição das imagens do paciente com a guia tomográfica. Os arquivos das imagens DICOM foram enviados ao Centro Tecnológico NeoGuide ${ }^{\circledR}$ (NEODENT, Curitiba, Brasil), onde foi realizada a instalação virtual dos implantes e componentes protéticos, tendo sido devolvido para ajustes e aprovação do projeto virtual por parte do cirurgião-dentista, para, então, proceder-se a confecção do guia prototipado (Figura 3).

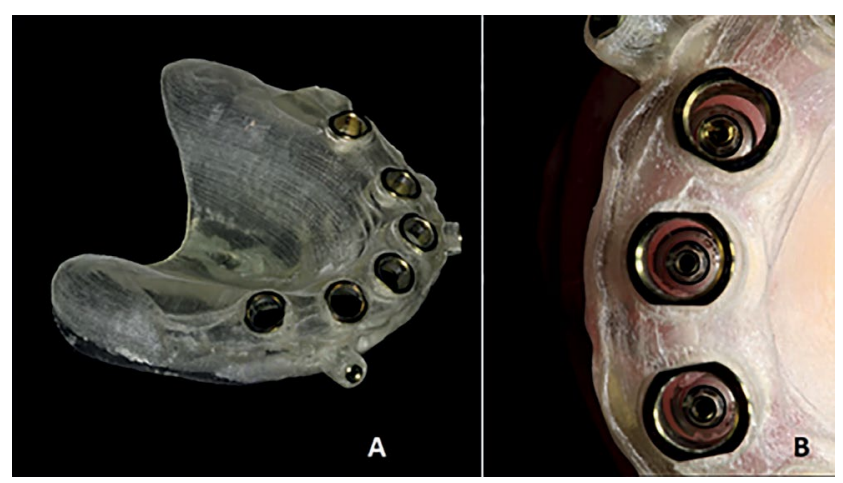

Figura 3 - Guia cirúrgico (NEOGUIDE ${ }^{\circledR}$, Curitiba, Brasil): A) Guia com seis anilhas e B) Neoguide sobre os modelos com minipilares

Fonte: elaboração dos autores.

\section{Procedimento cirúrgico}

Foram utilizadas as seguintes medicações pré-operatórias por via oral: quatro comprimidos de amoxicilina $(500 \mathrm{mg})$, cinquenta gotas de dipirona e dois comprimidos de dexametasona (4 mg), todos duas horas antes do procedimento. Da mesma forma, foi solicitado que a paciente utilizasse, antes do procedimento cirúrgico, bochechos com digluconato de clorexidina $0,12 \%$.

Após a antissepsia intrabucal e extrabucal e a desinfecção do guia cirúrgico prototipado, a paciente foi anestesiada com solução anestésica à base de cloridrato de mepivacaína $2 \%$ com epinefrina 1:100.000. Assim, posicionou-se o guia cirúrgico na boca da paciente, utilizando-se um registro de mordida em silicona de adição (Express XT ${ }^{\circledR}, 3 \mathrm{MESPE}$, Campinas, SP, Brasil) ancorado nos dentes inferiores, permitindo o posicionamento mais fiel e a estabilização por pinos retentores, inseridos em perfurações transversais sobre o tecido ósseo vestibular na maxila (Figura 4).

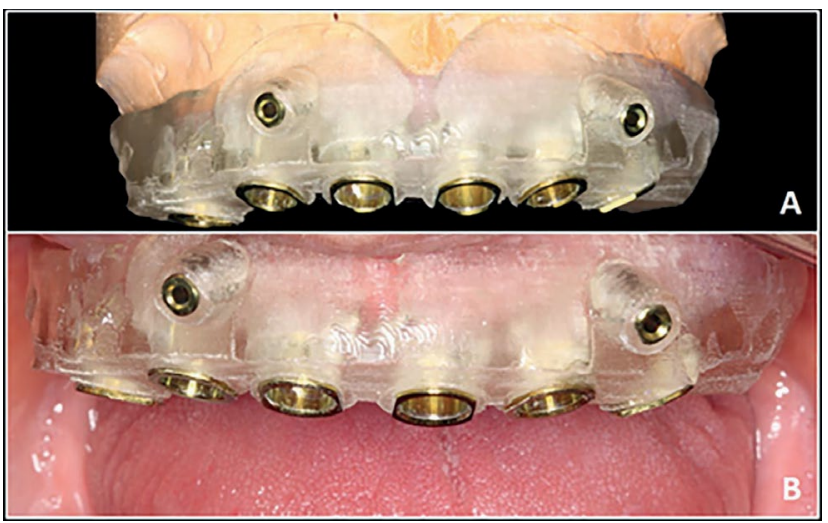

Figura 4 - Comparação entre a guia cirúrgica sobre o modelo de gesso $(A)$ e na boca $(B)$

Fonte: elaboração dos autores.

Um terceiro ponto de estabilização foi realizado na rafe palatina com a inserção de um parafuso de $1,5 \mathrm{~mm}$ x $10 \mathrm{~mm}$. Utilizando o kit cirúrgico Neogui$\mathrm{de}^{\circledR}$ (NEODENT, Curitiba, Brasil), a instrumentação cirúrgica baseou-se em um protocolo conforme tipo de osso e desenho do implante. Neste caso clínico, optou-se por implantes TITAMAX EX ${ }^{\circledR}$, todos de Ø3.5 x $13 \mathrm{~mm}$, com plataforma cone morse (NEODENT, Curitiba, Brasil), indicados para o osso tipo III e IV. A instrumentação cirúrgica foi realizada com sequência progressiva de diâmetros de broca 2.0 e 2.8 , em que a instalação final foi realizada com contra-ângulo 20:1 a 30 rpm. Após a instalação de cada um dos implantes, os parafusos estabilizadores de guia foram instalados, no intuito de não alterar a posição dos demais implantes que seriam colocados (Figura 5). 


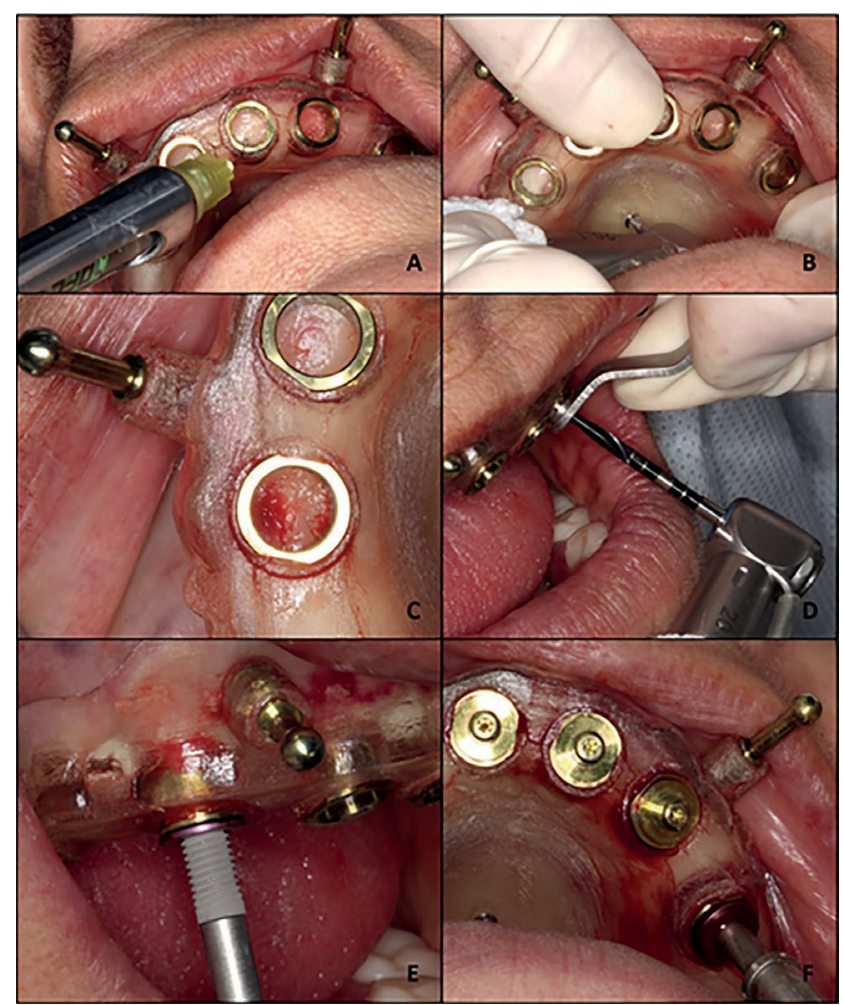

Figura 5 - Protocolo cirúrgico Neoguide: A) fixação dos pinos de estabilização e anestesia; $B$ ) estabilização na rafe palatina; C) anilhas da guia e perfurações; D) demais perfurações; E) instalação dos implantes; F) finalização do ato cirúrgico

Fonte: elaboração dos autores.

Com os implantes instalados, o guia cirúrgico prototipado foi removido e o torque de inserção e ISQ (quociente de estabilidade de implante) foram aferidos respectivamente com torquímetro e análise de frequência de ressonância (Osstell ${ }^{\circledR}$, Göteborg, Sweden). Devido aos torques baixos, todos menores de $20 \mathrm{~N}$ e ISQs também baixos, a carga imediata foi inviabilizada, sendo instalada uma prótese total convencional provisória, a mesma prótese utilizada nos procedimentos pré-operatórios. $\mathrm{O}$ aspecto pós-operatório final revela uma ferida cirúrgica minimamente traumática, em que apenas os orifícios de instalação do implante são visíveis, havendo a ausência de suturas, um mínimo edema e sangramento breve, compatíveis com o procedimento (Figura 6).

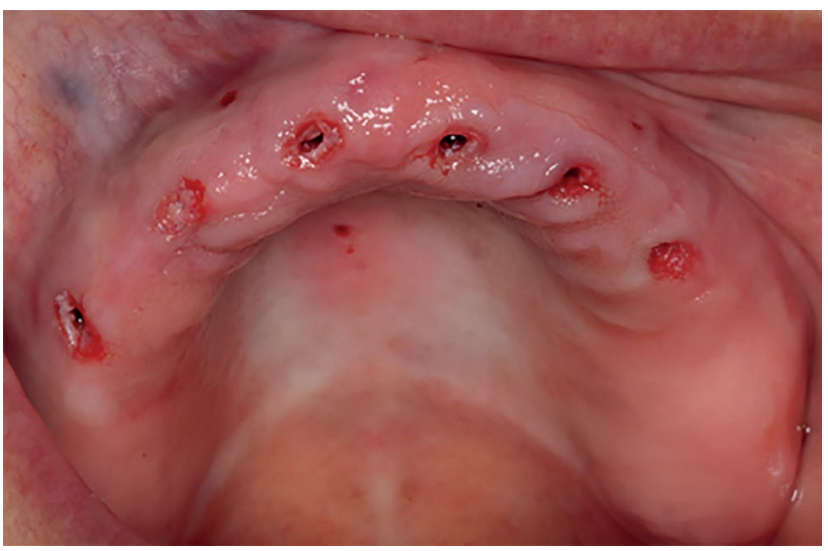

Figura 6 - Aspecto cirúrgico final após remoção do guia

Fonte: elaboração dos autores.
A paciente foi submetida a uma nova TCFC pós-operatória imediata para comparar o posicionamento virtual e real dos implantes, sendo as imagens sobrepostas, para observar possíveis desvios na posição (Figura 7).

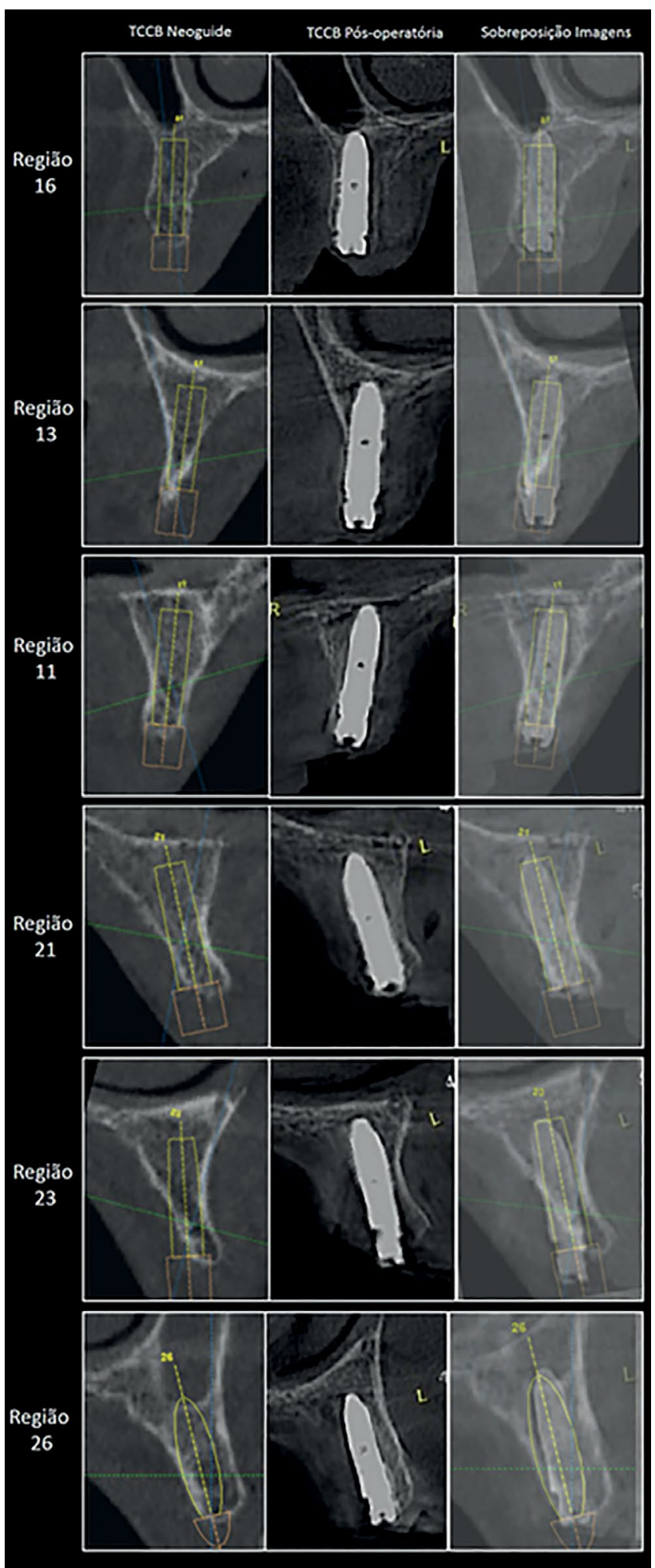

Figura 7 - Montagem dos exames de TCCB

Fonte: elaboração dos autores. 


\section{Discussão}

O planejamento tridimensional virtual com TCCB pré-operatória vem sendo cada vez mais utilizado no dia a dia do implantodontista, sobretudo pela facilidade da técnica, da diminuição de custos ao paciente e da constante atualização dos institutos de imaginologia quanto à qualidade dos exames ${ }^{1,3}$. Combinada a essas facilidades, a cirurgia sem retalho reduz a morbidade e o desconforto do paciente no pós-operatório imediato ${ }^{8}$. Como vantagens associadas à cirurgia virtualmente guiada, apresentam-se a colocação de implantes sem a realização de retalhos ${ }^{9}$, por meio de procedimentos cirúrgicos mais conservadores e cirurgias minimamente invasivas, que resultam em maior conforto para o paciente no trans e pós-operatório e em menor tempo total de tratamento ${ }^{3}$. Além disso, há ainda a precisão da técnica para instalação de implantes em regiões próximas a estruturas nobres (nervo alveolar inferior, forame mentoniano, fossa submandibular, forame nasopalatino, seio maxilar, etc.) ou em regiões com pouco espaço, em que a proximidade com as raízes dos dentes vizinhos seja um fator de risco no planejamento convencional ${ }^{1,5,10}$.

Algumas consultas pré-operatórias são indispensáveis para a realização de um adequado protocolo para cirurgia guiada, ainda mais, quando a confecção de uma prótese total nova deva ser feita para garantir uma condição estético-funcional ideal para o sucesso do caso ${ }^{3}$. Dessa forma, um tratamento pré-cirúrgico rígido e bem detalhado permite a economia de tempo no trans e pós-operatório, especialmente por se dispensar um segundo estágio cirúrgico, como também os procedimentos clínicos ou laboratoriais adicionais ${ }^{1,3,5}$. Quando comparado à técnica cirúrgica convencional de retalho aberto, tem-se um procedimento mais breve e objetivo, com uma morbidade significativamente menor ${ }^{3}$.

A instalação de implantes por meio da cirurgia guiada apresenta elevadas taxas de sobrevivência de implantes, que variam de $91 \%$ a $100 \%{ }^{2}$. Muito provavelmente, o elevado sucesso dessa técnica se deve às imagens tridimensionais da morfologia óssea obtidas com base nas imagens de TCCB, as quais permitem ao cirurgião visualizar, previamente à colocação do implante, o procedimento cirúrgico, garantindo o correto posicionamento dos implantes em áreas próximas a estruturas importantes, facilitando a reabilitação protética em condições adequadas $^{2-6}$. Todo esse contexto permite uma melhor previsibilidade de tratamento, um maior controle dos riscos, obtendo informações mais precisas da anatomia do paciente ${ }^{2}$.

A cirurgia guiada de implante melhora a qualidade tanto do procedimento cirúrgico quanto dos resultados restauradores, permitindo uma reabilitação segura e previsível em comparação com a cirurgia convencional ${ }^{10-15}$. Além disso, os idosos, pacientes clinicamente comprometidos e ansiosos, podem se beneficiar muito com a utilização desse protocolo cirúrgico ${ }^{5,6,8,11-13}$. Entre as vantagens para os pacientes, ressaltam-se a natureza minimamente invasiva desse tipo de procedimento, a precisão do posicionamento dos implantes cirurgicamente instalados, a previsibilidade em longo prazo, o menor desconforto pós-cirúrgico e a redução do tempo dispensado na cirurgia e na reabilitação protéti$\mathrm{ca}^{1,12,14,16}$. Em virtude do baixo travamento dos implantes $(20 \mathrm{~N})$ constatados no transcirúrgico deste caso clínico, a carga imediata que havia sido planejada foi cancelada, mantendo-se a utilização da nova prótese total, também utilizada para o planejamento virtual do caso. Porém, em situações em que haja um bom travamento inicial dos implantes, a carga imediata pode ser, tranquilamente, realizada, permitindo ao paciente a utilização de uma prótese fixa parafusada sobre esses implantes e, nesse caso, por se tratar de um protocolo superior, a ausência do palato de acrílico possibilita maior conforto fonético e alimentar por parte do paciente já durante o pós-cirúrgico.

Segundo Bornstein et al. ${ }^{10}$ (2014), os implantes instalados, utilizando a técnica de cirurgia guiada em acompanhamento longitudinal de 12 meses, demonstram uma taxa de sobrevivência média de $97,3 \%(\mathrm{n}=1.941)$, quando comparados à técnica convencional, em que as taxas de sucesso variaram de $93 \%$ a $98 \%$. Além disso, complicações sobre a qualidade pós-cirúrgica dos tecidos moles, infecção, deiscência de suturas e necrose dos tecidos perimplantares foram menores quando utilizada a técnica de cirurgia guiada. Muito provavelmente pela ausência de retalho e menor manipulação dos tecidos moles e duros ${ }^{6}$. O caso relatado apresentou sucesso de $100 \%$ no quesito osseointegração, sendo que a paciente relatou ausência de dor, edema, equimose ou outras alterações durante o período de pós-operatório.

A comparação das imagens realizadas no pré e pós-operatório deste caso clínico foi realizada da seguinte maneira: observando-se as regiões de inserção dos implantes em que a cirurgia virtual fora realizada, buscaram-se as sobreposições dos mesmos implantes inseridos durante $o$ ato cirúrgico. Esse procedimento foi realizado em software livre (UTHCSA Image Tool 3.0, EUA), após a sobreposição das imagens em escala 1:1 (virtual: real), capturadas por meio das imagens oferecidas pelo serviço de imaginologia em que a paciente foi encaminhada. Após a sobreposição das imagens virtuais com as da pós-instalação dos implantes, sugeriu-se um posicionamento muito semelhante, demonstrando a fidelidade e a confiabilidade da técnica, quando realizada respeitando-se, criteriosamente, todos os passos.

Uma das grandes indicações da cirurgia guiada é a precisão obtida no pós-operatório quando comparado com o planejamento virtualmente realizado no 
pré-cirúrgico ${ }^{1,3,5,9,12}$. Para os autores Verhamme et al. ${ }^{12}$ (2015), pequenas alterações verificadas quando da comparação do pré com o pós-operatório não são estatisticamente significativas ${ }^{12}$. Dessa forma, desvios médios obtidos com essa comparação no ponto de inserção dos implantes $(1,4 \mathrm{~mm} \pm 0,3$ a 3,7$)$, no seu ápice $(1,6 \mathrm{~mm} \pm 0,2$ a 3,7$)$ e desvios angulares $\left(3,0^{\circ}\right.$, intervalo: $\left.0,2-16^{\circ}\right)$, são substancialmente menores do que na cirurgia convencional $(2,8 \mathrm{~mm} \pm 0,3$ a 8,$3 ; 3,1 \mathrm{~mm} \pm 0,3$ a 7,5 e $9,1^{\circ} \pm 0,6$ a $\left.27,8^{\circ}\right)^{12}$.

Pode-se notar, pois, que esses resultados expressam a precisão obtida pela cirurgia guiada, mesmo que pequenas alterações possam ocorrer, o que é considerado importante para se elevar cada vez mais as taxas de sucesso, respeito a todas as etapas pré-operatórias, não só de confecção clinico-laboratorial de uma prótese total, funcionalmente, adequada, mas, também, de um bom exame imaginológico e de um planejamento bem elaborado ${ }^{1,3,12}$.

\section{Considerações finais}

De acordo com o caso clínico aqui apresentado, podemos concluir que a técnica apresentada permite um planejamento dos implantes com maior previsibilidade, refinadamente preciso, de modo que há redução substancial do tempo e do trauma cirúrgico convencionalmente dispendido, como também maior satisfação por parte do paciente pela menor morbidade envolvida.

\section{Abstract}

Introduction: In current Implantology, the accuracy of preoperative planning during both implant installation and prosthetic restoration is an essential prerequisite for the successful rehabilitation of the maxillomandibular complex. Thus, surgical guides for implant installations have become a valuable tool for accurately transferring the planning of implants position to the surgical site. Implants installation through surgery guided by prototyped guides presents high success rates, and its main characteristic is reducing operative time due to planning and previous virtual surgery. Objective: This study aimed to describe the technical steps of a guided flapless surgery in the edentulous maxilla of a patient by reporting a clinical case that compares virtual planning to the final placement of implants installed through cone beam tomography. Case Report: Female patient, 64 years old, wanting to install implants in edentulous maxilla, where there was indication and bone availability to perform guided surgery. Final Considerations: According to the clinical case presented, it may be concluded that this surgical technique allows better planning of implants, makes the surgical procedure more accurate and faster, and increases the predictability of results.

Keywords: Computer-assisted surgery. Dental implants. Implant-supported dental prosthesis.

\section{Referências}

1. De Vico G, Spinelli D, Bonino M, Schiavetti R, Pozzi A, Ottria L. Computer-assisted virtual treatment planning combined with flapless surgery and immediate loading in the rehabilitation of partial edentulies. Oral Implantol (Rome) 2012; 5(1):3-10.

2. Margonar R, Queiroz TP, Luvizuto ER, Betoni-Júnior W, Zocal EA. Mandibular rehabilitation using immediate implant loading after computer-guided surgery. J Craniofac Surg 2012; 23(2):e129-32.

3. Balshi SF, Wolfinger GJ, Balshi TJ. Surgical planning and prosthesis construction using computed tomography, $\mathrm{CAD} / \mathrm{CAM}$ technology, and the Internet for immediate loading of dental implants. J Esthet Restor Dent 2006; 18(6):312-23.

4. Flügge TV, Nelson K, Schmelzeisen R, Metzger MC. Threedimensional plotting and printing of an implant drilling guide: simplifying guided implant surgery. J Oral Maxillofac Surg 2013; 71(8):1340-6.

5. Van Assche N, Vercruyssen M, Coucke W, Teughels W, Jacobs R, Quirynen M. Accuracy of computer-aided implant placement. Clin Oral Implants Res 2012; 23 Suppl 6:112-23.

6. Orentlicher G, Abboud M. Guided surgery for implant therapy. Oral Maxillofac Surg Clin North Am 2011; 23(2):239-56.

7. Sicilia A, Botticelli D. Working Group 3. Computer-guided implant therapy and soft- and hard-tissue aspects. The Third EAO Consensus Conference 2012. Clin Oral Implants Res 2012; 23 Suppl 6:157-61.

8. Vercruyssen M, Cox C, Coucke W, Naert I, Jacobs R, Quirynen M. A randomized clinical trial comparing guided implant surgery (bone- or mucosa-supported) with mental navigation or the use of a pilot-drill template. J Clin Periodontol 2014; 41(7):717-23.

9. Rosenfeld AL, Mandelaris GA, Tardieu PB. Prosthetically directed implant placement using computer software to ensure precise placement and predictable prosthetic outcomes. Part 1: diagnostics, imaging, and collaborative accountability. Int J Periodontics Restorative Dent 2006; 26(3):215-21.

10. Bornstein MM, Al-Nawas B, Kuchler U, Tahmaseb A. Consensus statements and recommended clinical procedures regarding contemporary surgical and radiographic techniques in implant dentistry. Int J Oral Maxillofac Implants 2014; 29 Suppl:78-82.

11. Noharet R, Pettersson A, Bourgeois D. Accuracy of implant placement in the posterior maxilla as related to 2 types of surgical guides: a pilot study in the human cadaver. J Prosthet Dent 2014; 112(3):526-32.

12. Verhamme LM, Meijer GJ, Boumans T, de Haan AF, Bergé SJ, Maal TJ. A clinically relevant accuracy study of computer-planned implant placement in the edentulous maxilla using mucosa-supported surgical templates. Clin Implant Dent Relat Res 2015; 17(2):343-52.

13. Marquardt P, Witkowski S, Strub J. Three-dimensional navigation in implant dentistry. Eur J Esthet Dent 2007; 2(1):80-98.

14. Van Steenberghe D, Naert I, Andersson M, Brajnovic I, Van Cleynenbreugel J, Suetens P. A custom template and definitive prosthesis allowing immediate implant loading in the maxilla: a clinical report. Int $\mathrm{J}$ Oral Maxillofac Implants 2002 ; 17(5):663-70

15. Viegas VN, Dutra V, Pagnoncelli RM, de Oliveira MG. Transference of virtual planning and planning over biomedical prototypes for dental implant placement using guided surgery. Clin Oral Implants Res 2010; 21(3):290-5. 
16. Albiero AM, Benato R. Computer-assisted surgery and intraoral welding technique for immediate implant-supported rehabilitation of the edentulous maxilla: case report and technical description. Int J Med Robot 2015; DOI: 10.1002/ rcs.1715. [Epub ahead of print].

\section{Endereço para correspondência:}

Prof. Dr. Luciano Mayer

Rua Felipe Neri, 296/403

Bairro Auxiliadora

90440-150 Porto Alegre-RS

Telefone: (51) 3388-8452

E-mail: contato@clinicamayer.com.br

Recebido: 31/07/15. Aceito: 10/03/16. 